\title{
The issue of immunology in stem cell therapies: a pharmaceutical perspective
}

\author{
"...immunological privilege does not mean lack of immune response..."
}

Keywords: clinical trials $\bullet$ hESC $\bullet$ immune privilege $\bullet$ immune rejection $\bullet$ immunodeficient $\bullet$ iPS $\bullet$ MSC $\bullet$ mice $\bullet$ stem cells

As increasing numbers of stem cell therapies are entering clinical trials, the issue of the immunological consequences of these therapies has not kept pace with our knowledge of their cell biology. In simple terms, the immunological questions for stem cell derived therapies can be divided into three categories; the properties of the administered cells, the relative immune tolerance of the site of administration and the immune status of the host or patient.

\section{Immune privilege}

Various descriptions have been applied to stem cell therapies from immune privileged [1] to immune evasive or weakly immunogenic [2], but many of these characteristics are ascribed from in vitro assessments and how these results translate to the clinical setting is not clear. Many factors may be important in determining the susceptibility of transplanted cells to host immune rejection; allogeneic versus autologous cells, cell culture conditions, cell maturation, level of cellular manipulation, presence of inflammation and the immunological status of the patient.

Polymorphism within the MHC antigens is known to precipitate immune rejection. Expression of MHC class I is low in MSC therapies [3] and upregulated during human embryonic stem cells (hESC) differentiation, derived cell maturation and in the presence of inflammation [4]. This could result in increased susceptibility to immune rejection through recipient $\mathrm{T}$ lymphocyte allorecognition and rejection and this has been demonstrated in both in vitro (allogeneic) and in vivo (xenogeneic) studies [4]. Manipulating cells to downregulate their MHC expression is not without risk however, since this can increase susceptibility to cell killing by host natural killer (NK) cells.

One of the perceived benefits of developing induced pluripotent stem (iPS) cells was their increased likelihood of immune compatibility when given autologously following ex vivo culture to a patient. However, two sources of potential immunogenic antigens have been described that could lead to rejection even if the MHC is fully matched [5]. The first is the induction of neo-antigens as a result of genomic alterations during reprogramming [6]. The second is the observation that the process of reprogramming to pluripotency may lead to the expression of development genes required early in ontogeny. Such antigens are normally silenced before the emergence of the immune system and therefore the host $\mathrm{T}$ cells are not rendered tolerant [7].

Cell therapies may be purported, in some cases, as being administered to immunologically privileged sites. However, immunological privilege does not mean lack of immune response but rather the expression of local factors that act to prevent uncontrolled immunological reactions that could be catastrophic for the tissue in which they occur, such as the eye or brain. However, in the diseased state immunological privilege is often lost through breakdown of blood: tissue barriers (e.g., blood-retinal-barrier) and the administered cell therapies may be subject to the full force of the host immune system.

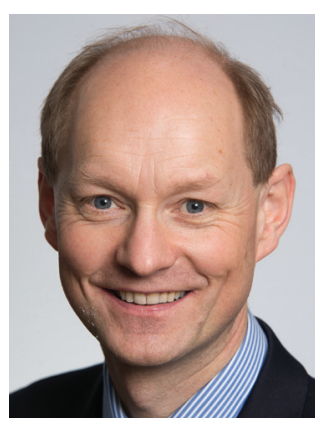

Richard Haworth *Author for correspondence: Safety Assessment, GlaxoSmithKline R\&D, Park Road, Ware, Herts., SG12 ODP, UK

richard.i.haworth@gsk.com

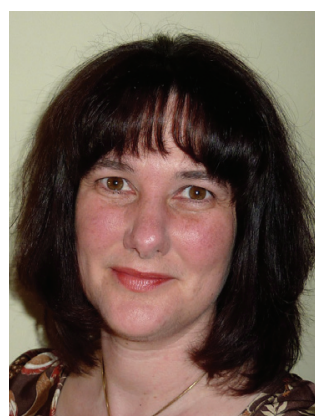

Michaela Sharpe

Cell Therapy Catapults Ltd, 12th Floor Tower Wing, Guys Hospital Great Maze Pond, London, SE1 9RT, UK 
An important question to consider early in any development program is whether the patient population is naturally immunosuppressed or will this be induced as part of the clinical approach? If the latter, will it be short term while the administered cells remain viable or does it need to be long term? Long-term immunosuppression is acceptable for life saving therapy but the side effects, including reduced immunosurveillance for tumor development, make this approach unacceptable for many of the chronic diseases being considered for cell therapy.

Therefore from the perspective of understanding the risk: benefit of any potential cell therapy product with a view to committing further investment, an improved understanding of the likely effects of any immune response on the safety and efficacy of a product will be critical [8].

\section{How to assess potential risk of immunogenicity or immune rejection}

Immune rejection in the clinical setting remains an important issue to be addressed. Loss of cells may result in limited efficacy and in certain circumstances the potential for serious adverse events. At the least, immune rejection limits the ability to repeat dose a cell therapy. For some indications, only limited duration of

\section{(6) RegMedNet}

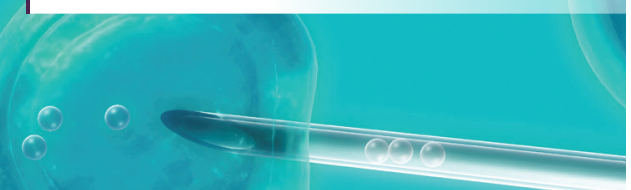

MAKE A DIFFERENCE

Find out how, join our online community today

Join today

www.RegMedNet.com cell survival is required since efficacy is dependent on a short-term trophic effect. However, the aim of many treatments is tissue or organ replacement which will require long-term cell survival and avoidance of host immune response.

Immune rejection remains difficult to address preclinically. Administration of the clinical product into an immune competent animal is a xenogeneic situation and any responses generated will not be predictive of the clinical setting. This applies not only to the use of rodents but also limits the utility of nonhuman primates. Use of severely immunodeficient or immunosuppressed animals, generally mice, are a practical solution to prolonging human cell survival to 'avoid' the effects of the animal immune system. These models allow certain safety risks, such as tumorigenesis of the administered cells to be evaluated, but cannot inform the risk of host rejection.

Species-specific cell products, for example, differentiated murine stem cells, designed as homologous cells to evaluate efficacy or safety, may allow prolonged cell survival but will not represent all aspects of the clinical product (e.g., cell surface markers) and species differences in the immune system may limit clinical extrapolation. An analogous situation arises with the preclinical safety testing of homologous monoclonal antibodies as a means of avoiding antidrug antibody development with fully human antibodies in animals. Humanized mouse models, which include aspects of the human immune system, may be an alternative approach. Remarkable progress has been achieved in humanized mouse models using NOD/SCID/ $\gamma_{c}$ [null], Rag1/2 [null] $\gamma_{c}$ [null] mice [9]. Several issues remain to be overcome, such as the rare differentiation of certain cell lineages from hematopoietic stem cells (HSC), immature differentiation and insufficient intercellular relationships (between animal and human cells) but these new mouse strains provide the potential for examining the risks of immune rejection.

An alternative approach is to perform a series of in vitro immunogenicity assessments on cells produced using the clinical manufacturing protocol, noting that their phenotype will, however, respond to their in vivo environment/niche. Cells should be assessed for MHC class I and MHC class II expression and cytokine/chemokine induction in presence and absence of proinflammatory cytokines that may be elevated in the target patient population [10]. Such studies can highlight potential risks such as upregulation of MHC II and co-stimulatory molecules (characteristics of APCs) but also potentially desirable expression of immunosuppressive molecules. Mixed lymphocyte reactions (using volunteer or patient peripheral blood mononuclear cells as responder cells and the product as stimulator 
cells) and susceptibility to NK-mediated cytotoxicity may also provide an in vitro assessment of risks of immune rejection.

\section{In the clinic}

Although the majority of clinical trials are for adult mesenchymal stem based therapies, the first pluripotent cell based therapies are now also being assessed. Has the risk of immune rejection been realized? A recent report has looked at the adventitial delivery of allogeneic bone marrow-derived adherent stem cells (MultiStem) in acute myocardial infarction [11]. No patients developed immune responses up to 4 months following treatment despite prestudy antibodies to MultiStem-expressed HLA antigens in two patients. More recently, it has been proposed that MSC therapies may have a limited window of therapeutic function due to rejection by the recipient immune system or to an inability to survive [12].

\section{"Immune rejection remains difficult to address preclinically.}

Preliminary data [13] for embryonic stem cell trials for macular degeneration (MD) have reported no transplant rejection. In this study, the immunosuppression regimen included low-dose tacrolimus and mycophenolate a week before the surgical procedure and continued for 6 weeks. After week 6 , the tacrolimus was discontinued and mycophenolate mofetil continued for an additional 6 weeks. Post-transplantation, there have been no signs of clinically significant inflammation. It is not yet known if the transplanted cells have reduced immunogenicity or whether they will undergo rejection without immunosuppression in the long term.

\section{The future}

With the first iPSC-derived cell therapy trials initiated in Japan (exudative MD; Riken Institute), there

\section{References}

1 Drukker M, Katz G, Urbach A et al. Characterization of the expression of MHC proteins in human embryonic stem cells. Proc. Natl Acad. Sci. USA 99(15), 9864-9869 (2002).

2 Ankrum JA, Ong JF, Karp JM. Mesenchymal stem cells: immune evasive, not immune privileged. Nat. Biotechnol. 32(3), 252-260 (2014).

3 Jacobs SA, Roobrouck VD, Verfaillie CM, Van Gool SW. Immunological characteristics of human mesenchymal stem cells and multipotent adult progenitor cells. Immunol. Cell Biol. 91(1), 32-39 (2013).

4 Pearl J.I, Wu J.C. The immunogenicity of ES cells and their differentiated progeny. In: The Immunological Barriers to Regenerative Medicine. Fairchild P.J. (Eds). Springer, New York, USA 37-48 (2013) is a need to better understand how reprogramming and expression of MHC and minor $\mathrm{HC}$ antigens can predict success following transplantation. In addition, the prospect now exists to build iPS banks with initiatives in a number of countries including the United Kingdom [14], and Japan [15]. Alternative areas of research include the generation of 'universal donor' stem cell lines [16] and the induction of immunological tolerance before treatment with allogeneic stem cell therapies [17]. As results from increasing numbers of MSC and PSC trials become available, the impact of any immune rejection on the safety and efficacy of these therapies will become clear.

\section{Summary}

Cautious approaches in the clinic are currently proposed, supported by relevant in vitro, in vivo and published data. Key to developing our understanding of the risks of immune rejection of stem cell based therapies will be the inclusion of immunological endpoints in clinical trials and the sharing of data. There is likely not a one-size-fits all strategy but one dependent on cell therapy, mode of action and disease indication.

\section{Acknowledgements}

We thank N Mount, C Maier and J Holder for their careful review.

\section{Financial \& competing interests disclosure}

R Haworth is employed by GlaxoSmithKline R\&D and M Sharpe is employed by Cell Therapy Catapults Ltd. The authors have no other relevant affiliations or financial involvement with any organization or entity with a financial interest in or financial conflict with the subject matter or materials discussed in the manuscript apart from those disclosed.

No writing assistance was utilized in the production of this manuscript.

5 Fairchild P. Interview: Immunogenicity: the elephant in the room for regenerative medicine? Interviewed by Alexandra Hemsley. Regen. Med. 8(1), 23-26 (2013).

6 Fu X. The immunogenicity of cells derived from induced pluripotent stem cells. Cell Mol. Immunol. 11(1), 14-16 (2014).

7 Zhao T, Zhang ZN, Rong Z, Xu Y. Immunogenicity of induced pluripotent stem cells. Nature 474(7350), 212-215 (2011).

8 Goldring CE, Duffy PA, Benvenisty N et al. Assessing the safety of stem cell therapeutics. Cell Stem Cell 8(6), 618-628 (2011).

9 Ito R, Takahashi T, Katano I, Ito M. Current advances in humanized mouse models. Cell Mol. Immunol. 9(3), 208-214 (2012). 
10 Okamura RM, Lebkowski J, Au M, Priest CA, Denham J, Majumdar AS. Immunological properties of human embryonic stem cell-derived oligodendrocyte progenitor cells. J. Neuroimmunol. 192(1-2), 134-144 (2007).

11 Penn MS, Ellis S, Gandhi S et al. Adventitial delivery of an allogeneic bone marrow-derived adherent stem cell in acute myocardial infarction: phase I clinical study. Circ. Res. 110(2), 304-311 (2012).

12 von BL, Batsis I, Moll G et al. Analysis of tissues following mesenchymal stromal cell therapy in humans indicates limited long-term engraftment and no ectopic tissue formation. Stem Cells 30 (7), 1575-1578 (2012).

13 Schwartz SD, Hubschman JP, Heilwell G et al. Embryonic stem cell trials for macular degeneration: a preliminary report. Lancet 379(9817), 713-720 (2012).
14 Cell Therapy Catapult. https://ct.catapult.org.uk/-/cell-therapy-catapult-roslin-cellsto-create-clinical-grade-stem-cells-to-accelerate-researchinto-new-treatments.

15 Cyranoski D. Stem-cell pioneer banks on future therapies. Nature 488(7410), 139 (2012).

16 Riolobos L, Hirata RK, Turtle CJ et al. HLA engineering of human pluripotent stem cells. Mol. Ther. 21(6), 1232-1241 (2013).

17 Pearl JI, Lee AS, Leveson-Gower DB et al. Short-term immunosuppression promotes engraftment of embryonic and induced pluripotent stem cells. Cell Stem Cell 8(3), 309-317 (2011). 